\title{
COVARIANCE FUNCTION AND ERGODICITY OF ASYMPTOTICALLY STATIONARY RANDOM FIELDS
}

\author{
V.V. ANH AND K.E. LUNNEY
}

\begin{abstract}
A class of second-order asymptotically stationary random fields is shown to contain the class of almost harmonisable random fields. A continuity theorem which leads to the spectral representation for the covariance function of asymptotically stationary random fields is established. A mean ergodic theorem for the fields is also given. When stationarity is assumed, the results reduce to the well-known corresponding theorems for stationary random fields.
\end{abstract}

\section{INTRODUCTION}

Stationary random processes and fields have played a prominent role in the analysis of $1 D$ and $2 D$ signals, and their theory has reached a mature state of development. On the other hand, understanding of nonstationary processes and fields is still fragmentary although their consideration seems more realistic in many important applications, particularly in image processing. There have been many attempts to extend the results for stationary processes to various classes of nonstationary processes (see Bhagavan [3] and Rao [10] for recent surveys). In particular, harmonisable processes introduced by Loeve [6] and asymptotically stationary processes studied by Parzen [8] and Kampe de Feriet and Frankiel [5] received much attention. The latter class, also named the class KF by Rao [9], is defined as follows.

Let $\{X(t),-\infty<t<\infty\}$ be a second order process with zero mean and covariance

$$
R(s, t)=E X(s) \overline{X(t)}
$$

$\{X(t)\}$ is said to be asymptotically stationary if the limit as $T \rightarrow \infty$ of

$$
R_{T}(k)=\frac{1}{T} \int_{|k| / 2}^{T-|k| / 2} R\left(s-\frac{k}{2}, s+\frac{k}{2}\right) d s
$$

exists for every $k \in \mathbf{R}$. The limit, denoted by $R(k)$, is defined to be the covariance function of the process.

Received 23 July 1990

Copyright Clearance Centre, Inc. Serial-fee code: 0004-9729/91 \$A2.00+0.00. 
In Bhagavan [1] the class $K F$ was studied in detail. An interesting result established in Bhagavan [1] is that the class of harmonisable processes is a subclass of the class $K F$. The spectral representation for the covariance function of processes in the class $K F$ was also given there. A mean ergodic theorem for the same processes was presented in Bhagavan [2]. In these studies, the fourth moment condition on $\{X(t)\}$ is not assumed. However, one of the following conditions was imposed in Bhagavan's investigation:

$C_{1}$ The real part of $R_{T}(k)$ is of bounded variation in a small closed interval $[0, \delta]$ including the origin;

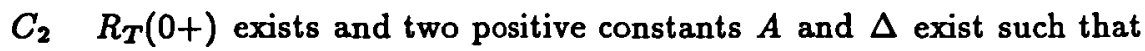

$$
\left|R_{T}(k)-R_{T}(0+)\right|<A|k|^{\Delta}
$$

for every $k$ in a small interval $[0, \delta]$ including the origin;

$C_{3} R_{T}(0+)$ exists and for $k$ in a small interval $[0, \delta]$ including the origin, the integral

$$
\int_{0+}^{6}\left(R_{T}(k)-R_{T}(0+)\right) d k / k
$$

converges absolutely.

As remarked by Bhagavan [1, p.60], the conditions $C_{1}, C_{2}, C_{3}$ do not seem to allow the results to cover the entire class of stationary processes as a particular case.

In this paper, we shall consider random fields. The class of asymptotically stationary random fields is defined. Its covariance function will then be studied. We shall establish a spectral representation for this covariance function under very mild conditions; in particular, the conditions $C_{1}, C_{2}, C_{3}$ corresponding to random fields will not be needed in the analysis, thus allowing the results to hold for stationary random fields.

Several examples of asymptotically stationary processes were given in Kempet de Feriet and Frenkiel [5] and Parzen [8]. Some practical application of the theory of these processes, particularly the computation of their spectrum, was demonstrated in Wolters [11] in an economic context. A particularly useful subclass consists of periodically correlated random fields. A study of the covariance function and spectrum of these fields through simulation is being carried out and will be reported elsewhere.

The assumptions required in the analysis are described in the next section, which also investigates the relation between almost harmonisable and asymptotically stationary random fields. The continuity theorem, and hence the spectral representation, for asymptotically stationary random fields is established in Section 3. Section 4 gives a mean ergodic theorem for these fields. 


\section{ASYMPTOTICALLY STATIONARY RANDOM FIELDS}

Consider a random field $\left\{X(t), t \in R^{n}\right\}$ on the probability space $(\Omega, \mathcal{F}, P)$ with mean zero and covariance $R(\mathrm{~s}, \mathrm{t})=E X(\mathrm{~s}) \overline{X(\mathrm{t})}$. We shall make the following assumptions:

(i) $X(t, \omega)$ is continuous in mean square;

(ii) $E|X(\mathbf{t})|^{2}<\infty, \mathbf{t} \in \mathbf{R}^{n}$;

(iii) $\int_{[a, b]} R(\mathrm{t}, \mathrm{t}) d \mathrm{t}<\infty$;

where $[a, b]=\left\{x \in R^{n}: a \leqslant x \leqslant b, a, b \in R^{n}\right\}$ and $d t$ is Lebesgue measure on $R^{n}$. Under condition (iii), Fubini's Theorem then implies that

$$
\begin{gathered}
\int_{[a, b]}|X(\mathrm{t})|^{2} d \mathrm{t}<\infty \quad \text { almost surely, } \\
E \int_{[a, b]}|X(\mathrm{t})|^{2} d \mathrm{t}<\infty \quad \text { and } \\
E \int_{[a, b]}|X(\mathrm{t})|^{2} d \mathrm{t}=\int_{[a, b]} E|X(\mathrm{t})|^{2} d \mathrm{t} .
\end{gathered}
$$

Let $\mathbf{k}=\left(k_{1}, \ldots, k_{n}\right), \mathbf{T}=\left(T_{1}, \ldots, T_{n}\right), T_{i} \geqslant 0, i=1, \ldots, n$.

We define

$$
R_{T}(k)=\prod_{j=1}^{n}\left(T_{j}\right)^{-1} \int_{a_{n}}^{b_{n}} \cdots \int_{a_{1}}^{b_{1}} R(\mathbf{t}, \mathbf{t}+\mathbf{k}) d \mathbf{t}
$$

where

$$
\begin{aligned}
& a_{i}= \begin{cases}0, & 0 \leqslant k_{i} \leqslant T_{i}, \\
\left|k_{i}\right|, & -T_{i} \leqslant k_{i} \leqslant 0,\end{cases} \\
& b_{i}= \begin{cases}T_{i}-k_{i}, & 0 \leqslant k_{i} \leqslant T_{i}, \\
T_{i}, & -T_{i} \leqslant k_{i} \leqslant 0,\end{cases}
\end{aligned}
$$

$i=1,2, \ldots, n$, and

$$
F_{T}(s)=\prod_{j=1}^{n}\left(T_{j}\right)^{-1} \int_{(-\infty, s]} \int_{[0, T]} \int_{[0, T]} R(\mathrm{t}, \mathrm{u}) e^{-i(\mathbf{u}-\mathrm{t}, \mathrm{v})} d \mathrm{t} d \mathbf{u} d \mathrm{v} .
$$

The random field $\left\{X(t), t \in R^{n}\right\}$ is said to be asymptotically stationary if the limit as $T_{j} \rightarrow \infty, j=1, \ldots, n$, of $R_{T}(\mathbf{k})$ exists for every $\mathbf{k} \in \mathbf{R}^{n}$. The limit, denoted by $R(\mathbf{k})$, is defined to be the covariance function of the random field. The class of asymptotically stationary random fields is denoted by $\mathcal{A}$. 
In the following, unless written out completely, the simpler notation lim will be used to mean $\lim _{\substack{T_{j} \rightarrow \infty \\ j=1, \ldots, n}}$

We first note that if $\{X(t)\}$ is stationary, then $R(t, t+k)=R(k)$. It then follows readily that $\lim _{T \rightarrow \infty} R_{T}(\mathbf{k})=R(\mathbf{k})$.

Thus $\mathcal{A}$ covers the class of stationary random fields. We next define the class of almost harmonisable random fields, following Rao [9].

Let $g(., \lambda)$ be an almost periodic function of $\mathbf{R}^{n}$ for almost all $\lambda \in \mathbf{R}^{n}$ (see the Appendix). A zero mean random field $\{X(t)\}$ is said to be almost harmonisable if its covariance function can be represented as

$$
R(\mathrm{~s}, \mathrm{t})=\int_{\mathbf{R}^{n}} \int_{\mathbf{R}^{n}} g(\mathbf{s}, \lambda) \overline{g(t, \mu)} \gamma(d \lambda, d \mu),
$$

where $\gamma(d \lambda, d \mu)$ is of bounded variation on each finite domain of $\mathbf{R}^{n} \times \mathbf{R}^{n}$. The class of almost harmonisable random fields is denoted by $\mathcal{H}$. It should be noted that, in the scalar case $(n=1)$, this is a subclass of Cramer's class $C$, where $\{g(., \lambda)\}$ is a family such that the integral of (2.2) exists. It is clear that $\mathcal{H}$ contains the class of harmonisable random fields, where $g(., \lambda)$ takes the special form $g(t, \lambda)=e^{i(t, \lambda)}$. We now want to show that

$$
\mathcal{H} \subset \mathcal{A}
$$

In fact, using (2.2) in $R_{T}(\mathbf{k})$, we get

$$
R_{T}(\mathbf{k})=\frac{1}{T_{1} \ldots T_{n}} \int_{a_{n}}^{b_{n}} \ldots \int_{a_{1}}^{b_{1}} \int_{\mathbf{R}^{n}} \int_{\mathbf{R}^{n}} g(t, \lambda) \overline{g(\mathbf{t}+\mathbf{k}, \mu)} \gamma(d \lambda, d \mu) d t .
$$

An application of Fubini's theorem yields

$$
R_{T}(\mathbf{k})=\int_{\mathbf{R}^{n}} \int_{\mathbf{R}^{n}} \frac{1}{T_{1} \ldots T_{n}} \int_{a_{n}}^{b_{n}} \ldots \int_{a_{1}}^{b_{1}} g(t, \lambda) \overline{g(t+k, \mu)} d t \gamma(d \lambda, d \mu) .
$$

In view of result (A1) of the Appendix, we have that

$$
\begin{gathered}
\lim _{\substack{T_{i} \rightarrow \infty \\
i=1, \ldots, n}} \prod_{i=1}^{n} \frac{T_{i}-\left|k_{i}\right|}{T_{i}} \lim _{\substack{T_{j} \rightarrow \infty \\
j=1, \ldots, n}} \prod_{j=1}^{n} \frac{1}{T_{j}-\left|k_{j}\right|} \int_{a_{n}}^{b_{n}} \ldots \int_{a_{1}}^{b_{1}} g(\mathbf{t}, \lambda) \overline{g(t+k, \mu)} d t \\
=M(g(\mathbf{t}, \lambda) \overline{g(\mathbf{t}+\mathbf{k}, \mu)} ; \mathbf{k}, \lambda, \mu) .
\end{gathered}
$$

It now follows that

$$
\lim _{T \rightarrow \infty} R_{T}(\mathbf{k})=\int_{\mathbf{R}^{n}} \int_{\mathbf{R}^{n}} M(g(\mathbf{t}, \lambda) \overline{g(\mathbf{t}+\mathbf{k}, \mu)} ; \mathbf{k}, \lambda, \mu) \gamma(d \lambda, d \mu)
$$

by the dominated convergence theorem, and (2.3) is obtained. 
REMARK. The result that harmonisable processes are asymptotically stationary was first proved by Bhagavan [1] using a different argument. The above approach, which is based on the mean value theorem for almost periodic functions, is due to Rao [9].

\section{A CONTINUITY THEOREM}

We first establish several results for $R_{T}(\mathrm{k})$ and $F_{T}(\mathrm{~s})$. It follows directly from its definition that $R_{T}(\mathbf{k})$ is Hermitian symmetric, that is,

$$
R_{T}(-\mathrm{k})=\overline{R_{T}(\mathrm{k})}, \mathrm{T}>0,-\mathrm{T} \leqslant \mathrm{k} \leqslant \mathrm{T} .
$$

Also, under the assumption that the random field $\{X(t)\}$ is continuous in mean square, it can be shown via the mean value theorem that $R_{T}(\mathbf{k})$ is a continuous function of $\mathbf{k}$ in $[-\mathbf{T}, \mathbf{T}]$.

From definition, we get $\lim _{s_{i} \rightarrow-\infty} F_{T}(\mathrm{~s})=0$ for some $s_{i}$. For $\mathrm{s}_{1}<\mathrm{s}_{2}$,

$$
\begin{aligned}
& F_{\mathrm{T}}\left(\mathrm{s}_{2}\right)-F_{\mathbf{T}}\left(\mathrm{s}_{1}\right)=\prod_{j=1}^{n}\left(T_{j}\right)^{-1} \int_{\left[s_{1}, s_{2}\right]} \int_{[0, T]} \int_{[0, T]} R(\mathrm{t}, \mathrm{u}) e^{-i(\mathrm{u}-\mathrm{t}, \mathrm{v})} d \mathrm{t} d \mathrm{u} d \mathrm{v} \\
& =\prod_{j=1}^{n}\left(T_{j}\right)^{-1} \int_{\left[\varepsilon_{1}, s_{2}\right]} E\left[\int_{[0, T]} X(\mathrm{t}) e^{i(\mathrm{t}, \mathrm{v})} d \mathrm{t} \int_{[0, T]} \overline{X(\mathrm{u})} e^{-i(\mathrm{u}, \mathrm{v})} d \mathrm{u}\right] d \mathrm{v} \quad \text { using }(2.1) \\
& =\prod_{j=1}^{n}\left(T_{j}\right)^{-1} \int_{\left[0_{1}, s_{2}\right]} E\left|\int_{[0, T]} X(\mathrm{t}) e^{i(\mathrm{t}, \mathrm{v})} d \mathrm{t}\right|^{2} d \mathrm{v} \geqslant 0 .
\end{aligned}
$$

Thus $F_{\mathrm{T}}(s)$ is non-negative and non-decreasing.

Lemma 1. $\left|R_{T}(\mathbf{k})\right| \leqslant R_{T}(0)$ for $\mathbf{k} \in \mathbf{R}^{n}$ and $T_{j}, j=1, \ldots, n$, sufficiently large.

PRoOF: We first prove that, for $k \in \mathbf{R}^{n}$,

$$
\begin{aligned}
\lim _{\substack{T_{j} \rightarrow \infty \\
j=1, \ldots, n}} \prod_{j=1}^{n}\left(T_{j}\right)^{-1} & \int_{a_{n}}^{b_{n}} \ldots \int_{a_{1}}^{b_{1}} E|X(\mathbf{t}+\mathbf{k})|^{2} d \mathrm{t} \\
& =\lim _{\substack{T_{j} \rightarrow \infty \\
j=1, \ldots, n}} \prod_{j=1}^{n}\left(T_{j}\right)^{-1} \int_{a_{n}}^{b_{n}} \ldots \int_{a_{1}}^{b_{1}} E|X(\mathrm{t})|^{2} d \mathrm{t} .
\end{aligned}
$$

In fact, by a change of variables,

$$
\prod_{j=1}^{n}\left(T_{j}\right)^{-1} \int_{a_{n}}^{b_{n}} \ldots \int_{a_{1}}^{b_{1}}|X(t+k)|^{2} d t=\prod_{j=1}^{n}\left(T_{j}\right)^{-1} \int_{a_{n}-k_{n}}^{b_{n}-k_{n}} \cdots \int_{a_{1}-k_{1}}^{b_{1}-k_{1}}|X(t)|^{2} d t .
$$


Using the same argument as in the proof of result (A1) in the Appendix will give (3.3). Next, we have

$$
\begin{aligned}
\left|R_{\mathrm{T}}(\mathbf{k})\right| & =\left|\prod_{j=1}^{n}\left(T_{j}\right)^{-1} E \int_{a_{n}}^{b_{n}} \cdots \int_{a_{1}}^{b_{1}} X(\mathrm{t}) \overline{X(\mathrm{t}+\mathrm{k})} d \mathrm{t}\right| \text { using }(2.1) \\
& \leqslant \prod_{j=1}^{n}\left(T_{j}\right)^{-1}\left(\int_{a_{n}}^{b_{n}} \cdots \int_{a_{1}}^{b_{1}} E|X(\mathrm{t})|^{2} d \mathrm{t}\right)^{1 / 2}\left(\int_{a_{n}}^{b_{n}} \cdots \int_{a_{1}}^{b_{1}} E|X(\mathrm{t}+\mathrm{k})|^{2} d \mathrm{t}\right)^{1 / 2} \\
& \text { using Cauchy-Schwarz's inequality } \\
& =\prod_{j=1}^{n}\left(T_{j}\right)^{-1}\left(\int_{a_{n}}^{b_{n}} \cdots \int_{a_{1}}^{b_{1}} E|X(\mathrm{t})|^{2} d \mathrm{t}\right) \\
& \leqslant \prod_{j=1}^{n}\left(T_{j}\right)^{-1} \int_{0}^{T_{n}} \cdots \int_{0}^{T_{1}} E|X(\mathrm{t})|^{2} d \mathrm{t} \\
& =R_{T}(0) .
\end{aligned}
$$

Thus, $\left|R_{T}(\mathbf{k})\right| \leqslant R_{T}(0)$.

LEMma 2. $F_{T}(\infty)=(2 \pi)^{n} R_{T}(0)$ for $T_{j}, j=1, \ldots, n$, sufficiently large.

Proof: A change of variables $(t, u) \rightarrow(k, t)$ with $k=u-t$ gives, using the definition of $\boldsymbol{F}_{\mathbf{T}}(\mathrm{s})$, that

$$
\begin{aligned}
F_{\mathbf{T}}(\mathbf{s}) & =\prod_{j=1}^{n}\left(T_{j}\right)^{-1} \int_{(-\infty, \text { d] }} \int_{[-T, T]} \int_{a_{n}}^{b_{n}} \cdots \int_{a_{1}}^{b_{1}} R(\mathbf{t}, \mathbf{t}+\mathbf{k}) e^{-i(k, v)} d t d \mathbf{k} d \mathbf{v} \\
& =\int_{(-\infty, \text {, }]} \int_{[-T, T]} R_{T}(\mathbf{k}) e^{-i(k, v)} d \mathbf{k} d \mathbf{v} \\
& =\int_{(-\infty, \text { s] }} \int_{\mathbf{R}^{n}} R_{T}^{*}(\mathbf{k}) e^{i(\mathbf{k}, \mathbf{v})} d \mathbf{k} d \mathbf{v}
\end{aligned}
$$

where

$$
R_{\mathbf{T}}^{*}(\mathbf{k})= \begin{cases}R_{T}(\mathbf{k}), & -\mathbf{T} \leqslant \mathbf{k} \leqslant \mathbf{T} \\ 0, & \text { otherwise }\end{cases}
$$


For $\mu>0$, we then get

$$
\begin{aligned}
F_{\mathbf{T}}(\mu)-F_{\mathbf{T}}(-\mu)= & \int_{[-\mu, \mu]} \int_{\mathbf{R}^{n}} R_{\mathbf{T}}^{*}(\mathbf{k}) e^{-i(k, v)} d \mathbf{k} d \mathbf{v} \\
= & \int_{\mathbf{R}^{n}} R_{\mathbf{T}}^{*}(\mathbf{k}) \int_{[-\mu, \mu]} e^{-i(k, v)} d \mathbf{v} d \mathbf{k} \\
& \quad \text { using Fubini's Theorem, since } \mathbf{R}_{\mathbf{T}}(\mathbf{k}) \text { is continuous on [-T, T], } \\
= & \int_{\mathbf{R}^{n}} R_{\mathbf{T}}^{*}(\mathbf{k}) \prod_{j=1}^{n} \int_{-\mu_{j}}^{\mu_{j}} e^{-i k_{j} v_{j}} d v_{j} d \mathbf{k} \\
= & 2^{n} \int_{\mathbf{R}^{n}} R_{\mathbf{T}}^{*}(\mathbf{k}) \prod_{j=1}^{n} \frac{\sin \mu_{j} k_{j}}{k_{j}} d \mathbf{k} .
\end{aligned}
$$

It follows that, for $s>0$,

$$
\begin{aligned}
\prod_{j=1}^{n}\left(s_{j}\right)^{-1} & \int_{[0, s]}\left(F_{\mathbf{T}}(\mu)-F_{\mathbf{T}}(-\mu)\right) d \mu=2^{n} \prod_{j=1}^{n}\left(s_{j}\right)^{-1} \int_{[0, \Omega]} \int_{\mathbf{R}^{n}} R_{\mathbf{T}}^{*}(\mathbf{k}) \frac{\sin \mu_{j} k_{j}}{k_{j}} d \mathbf{k} d \mu \\
& =\prod_{j=1}^{n} 2^{n}\left(s_{j}\right)^{-1} \int_{\mathbf{R}^{n}} R_{\mathbf{T}}^{*}(\mathbf{k}) \int_{0}^{s_{j}} \frac{\sin \mu_{j} k_{j}}{k_{j}} d \mu_{j} d \mathbf{k} \quad \text { by Fubini's Theorem } \\
& \leqslant \prod_{j=1}^{n} 2^{n}\left(s_{j}\right)^{-1} \max _{k}\left|R_{\mathbf{T}}(\mathbf{k})\right| \int_{-\infty}^{\infty} \frac{1-\cos s_{j} k_{j}}{k_{j}^{2}} d k_{j} \\
& =\prod_{j=1}^{n}(2 \pi)^{n} \max _{k}\left|R_{\mathbf{T}}(\mathbf{k})\right| \int_{-\infty}^{\infty} \frac{\sin s_{j} k_{j}}{\pi k_{j}} d k_{j} \\
& =(2 \pi)^{n} \max _{k}\left|R_{\mathbf{T}}(\mathbf{k})\right| .
\end{aligned}
$$

With the change of variables $\mu_{j}=s_{j} \alpha_{j}, j=1, \ldots, n$, we have

$$
\begin{aligned}
& \prod_{j=1}^{n}\left(s_{j}\right)^{-1} \int_{[0, \Omega]}\left[F_{\mathrm{T}}(\mu)-F_{\mathrm{T}}(-\mu)\right] d \mu \\
& \quad=\int_{0}^{1} \ldots \int_{0}^{1}\left[F_{\mathrm{T}}\left(s_{1} \alpha_{1}, \ldots, s_{n} \alpha_{n}\right)-F_{\mathrm{T}}\left(-s_{1} \alpha_{1}, \ldots,-s_{n} \alpha_{n}\right)\right] d \alpha_{1} \ldots d \alpha_{n} .
\end{aligned}
$$

Thus, for $\mu>0$,

$$
\begin{aligned}
\int_{[0,1]}\left[F_{\mathbf{T}}(\mu)-F_{\mathbf{T}}(-\mu)\right] d \alpha & \leqslant(2 \pi)^{n} \max _{k}\left|R_{\mathbf{T}}(\mathbf{k})\right| \\
& =(2 \pi)^{n} R_{\mathrm{T}}(0)
\end{aligned}
$$

using Lemma 1. 
Since $F_{\mathrm{T}}(s)$ is non-decreasing, the integral on the LHS of (3.4) is a non-decreasing function of $s$. It follows that

$$
\lim _{s \rightarrow \infty} \ldots \lim _{s \rightarrow \infty}\left(F_{\mathrm{T}}(\mathrm{s})-F_{\mathrm{T}}(-\mathrm{s})\right) \text { exists. }
$$

We then have for $T_{j}, j=1, \ldots, n$, sufficiently large, that

$$
\begin{aligned}
& F_{\mathrm{T}}(\infty)=\lim _{\substack{\mathfrak{c}_{j} \rightarrow \infty \\
j=1, \ldots, n}} \prod_{j=1}^{n}\left(\frac{2^{n}}{s_{j}}\right) \int_{\mathbf{R}^{n}} R_{\mathrm{T}}^{*}(\mathbf{k}) \frac{1-\cos s_{j} k_{j}}{k_{j}^{2}} d \mathbf{k} \\
& =\lim _{\substack{j \rightarrow \infty \\
j=1, \ldots, n}} \prod_{j=1}^{n} 2^{n} \int_{\mathrm{R}^{n}} R_{\mathbf{T}}^{*}(\ell) \frac{1-\cos k_{j}}{k_{j}^{2}} d \mathbf{k} \\
& \text { where } \ell_{j}=\frac{k_{j}}{s_{j}}, j=1, \ldots, n \\
& =(2 \pi)^{n} R_{\mathrm{T}}(0)+\lim _{\substack{\boldsymbol{s}_{j} \rightarrow \infty \\
j=1, \ldots, n}} 2^{n} \prod_{j=1}^{n} \int_{\mathbf{R}^{n}}\left(R_{\mathrm{t}}^{*}(\ell)-R_{\mathbf{T}}(0)\right) \frac{1-\cos k_{j}}{k_{j}^{2}} d \mathbf{k} .
\end{aligned}
$$

Thus, for any $\varepsilon>0$ and for $s_{j}, j=1, \ldots, n$, sufficiently large,

$$
\begin{aligned}
& \left|F_{\mathrm{T}}(\infty)-(2 \pi)^{n} R_{\mathrm{T}}(0)\right| \\
& \quad \leqslant \varepsilon+2^{n+1} R_{\mathrm{T}}(0) \int_{-\infty}^{\infty} \ldots\left[\left(\int_{-\infty}^{-e_{1}^{1 / 2}}+\int_{e_{1}^{1 / 2}}^{\infty}\right) \frac{1-\cos k_{1}}{k_{1}^{2}} d k_{1}\right] \prod_{j=2}^{n} \frac{1-\cos k_{j}}{k_{j}^{2}} d k_{j} \\
& +2^{n} \int_{-\infty}^{\infty} \cdots\left[\max _{\left|\ell_{1}\right|<\ell_{1}^{-1 / 2}}\left|R_{\mathrm{T}}\left(\ell_{1}, \ldots, \ell_{n}\right)-R_{\mathrm{T}}(0)\right| \int_{-e_{1}^{1 / 2}}^{\alpha_{1}^{1 / 2}} \frac{1-\cos k_{1}}{k_{1}^{2}}\right] \prod_{j=2}^{n} \frac{1-\cos k_{j}}{k_{j}^{2}} d k_{j} .
\end{aligned}
$$

Repeating the argument for the remaining components of $k$, we will find that

$$
\begin{aligned}
\left|F_{\mathrm{T}}(\infty)-(2 \pi)^{n} R_{\mathrm{T}}(0)\right| & \leqslant \varepsilon+2^{n} \max _{\left|\ell_{j}\right|<\alpha_{j}^{-1 / 2}, j=1, \ldots, n}\left|R_{\mathrm{T}}\left(\ell_{1}, \ldots, \ell_{n}\right)-R_{\mathrm{T}}(0)\right| \\
& \times \prod_{j=1}^{n}\left(\int_{-\alpha_{j}^{1 / 2}}^{\alpha_{j}^{1 / 2}} \frac{1-\cos k_{j}}{k_{j}^{2}} d k_{j}\right)+\mathcal{R},
\end{aligned}
$$

where $\mathcal{R}$ involves terms with integrals of the form $\left(\int_{-\infty}^{-\infty} j_{j}^{1 / 2}+\int_{s_{j}^{1 / 2}}^{\infty}\right)$. These integrals vanish as $s_{j}, j=1, \ldots, \mathrm{n}$, can be chosen arbitrarily large. Also, since $\varepsilon$ can be arbitrarily small, we get

$$
\left|F_{\mathbf{T}}(\infty)-(2 \pi)^{n} R_{\mathbf{T}}(0)\right| \leqslant(2 \pi)^{n} \limsup _{|\ell| \rightarrow 0}\left|R_{\mathbf{T}}(\ell)-R_{\mathbf{T}}(0)\right|
$$


As $R_{\mathbf{T}}(\ell)$ is continuous at $\ell=0$, we obtain immediately that $F_{\mathbf{T}}(\infty)=$ $(2 \pi)^{n} R_{\mathbf{T}}(0)$.

We will now establish the required continuity theorem.

Theorem 1. For a random field $\left\{X(\mathrm{t}), t \in \mathrm{R}^{n}\right\}$ under conditions (i) - (iii), and assuming that $\lim _{T \rightarrow \infty} R_{\mathrm{T}}(0)$ exists finitely, we have $\lim _{T \rightarrow \infty} F_{\mathrm{T}}(\mathrm{s})=F(\mathrm{~s})$ at every continuity point of $F(\mathrm{~s})$, a non-negative, non-decreasing and bounded function on $\mathrm{R}^{n}$, if and only if $\lim _{\mathrm{T} \rightarrow \infty} R_{\mathrm{T}}(\mathrm{k})=R(\mathrm{k})$, a function which is continuous at the origin. Furthermore, $R(k)$ has the representation

$$
R(\mathbf{k})=\left(\frac{1}{2 \pi}\right)^{n} \int_{R^{n}} e^{i(k, s)} d F(\mathrm{~s})
$$

Proof: As in the proof of Lemma 2, we have

$$
F_{\mathrm{T}}(\mathrm{s})=\int_{(-\infty, \mathrm{d}]} \int_{\mathbf{R}^{n}} R_{\mathrm{T}}^{*}(\mathrm{k}) e^{-i(\mathrm{k}, \mathrm{v})} d \mathrm{k} d \mathrm{v}
$$

For $\mathbf{T}^{1}>0$, define

$$
I_{\mathbf{T}^{1}}=\left(\frac{1}{2 \pi}\right)^{n} \int_{\left[-T^{1}, T^{1}\right]} e^{i(u, s)} d F_{\mathbf{T}}(s)
$$

Then, from (3.5),

$$
\begin{aligned}
I_{\mathbf{T}^{1}} & =\left(\frac{1}{2 \pi}\right)^{n} \int_{\left[-T^{1}, T^{1}\right]} \int_{\mathbf{R}^{n}} R_{T}^{*}(\mathbf{k}) e^{i(\mathrm{u}-\mathrm{k}, \mathrm{s})} d \mathbf{k} d \mathbf{s} \\
& =\left(\frac{1}{2 \pi}\right)^{n} \int_{\mathbf{R}^{n}} R_{\mathrm{T}}^{*}(\mathbf{k}) \int_{\left[-T^{1}, T^{1}\right]} e^{i(\mathbf{u}-\mathbf{k}, \mathrm{s})} d \mathbf{s} d \mathbf{k}
\end{aligned}
$$

using Fubini's theorem as $R_{T}(\mathrm{k})$ is continuous on $\left[-T_{1}, T\right]$

$$
\begin{aligned}
& =\prod_{j=1}^{n}\left(\frac{1}{\pi}\right)^{n} \int_{\mathbf{R}^{n}} R_{\mathrm{T}}^{*}(\mathbf{k}) \frac{\sin \left(u_{j}-k_{j}\right) T_{j}^{1}}{\left(u_{j}-k_{j}\right)} d \mathbf{k} \\
& =\prod_{j=1}^{n}\left(\frac{1}{\pi}\right)^{n} \int_{-\infty}^{\infty} \ldots \int_{-\infty}^{\infty} R_{T}^{*}\left(u_{1}-\frac{\tau_{1}}{T_{1}^{1}}, \ldots, u_{n}-\frac{\tau_{n}}{T_{n}^{1}}\right) \frac{\sin \tau_{j}}{\tau_{j}} d \tau_{1}, \ldots, d \tau_{n}
\end{aligned}
$$
using the change of variables $\tau_{j}=\left(u_{j}-k_{j}\right) T_{j}^{1}, j=1, \ldots, n$.

Lemma 2 yields that

$$
\int_{\mathbf{R}^{n}} d F_{\mathbf{T}}(\mathbf{s})=F_{\mathbf{T}}(\infty)-F_{\mathbf{T}}(-\infty)=(2 \pi)^{n} R_{\mathbf{T}}(0)<\infty
$$


Thus the dominated convergence theorem implies that

$$
\lim _{T^{1} \rightarrow \infty}\left(\frac{1}{2 \pi}\right)^{n} \int_{\left[-T^{1}, T^{1}\right]} e^{i(u, s)} d F_{T}(s)=\left(\frac{1}{2 \pi}\right)^{n} \int_{R^{n}} e^{i(u, s)} d F_{T}(s) .
$$

In view of the dominated convergence theorem and the continuity of $R_{T}(k)$ on $[-T, T]$ we have for $T_{j}, j=1, \ldots, n$, sufficiently large that

$$
\begin{aligned}
\lim _{T^{1} \rightarrow \infty} & \left(\frac{1}{\pi}\right)^{n} \prod_{j=1}^{n} \int_{-\infty}^{\infty} \cdots \int_{-\infty}^{\infty} R_{\mathrm{T}}^{*}\left(u_{1}-\frac{\tau_{1}}{T_{1}^{1}}, \ldots, u_{n}-\frac{\tau_{n}}{T_{n}^{1}}\right) \frac{\sin \tau_{j}}{\tau_{j}} d \tau_{1} \ldots d \tau_{n} \\
& =\left(\frac{1}{\pi}\right)^{n} \prod_{j=1}^{n} \int_{-\infty}^{\infty} \cdots \int_{-\infty}^{\infty} R_{\mathrm{T}}^{*}\left(u_{1}, \ldots, u_{n}\right) \frac{\sin \tau_{j}}{\tau_{j}} d \tau_{1} \ldots d \tau_{n} \\
& =R_{\mathrm{T}}(\mathbf{u}) .
\end{aligned}
$$

Thus it follows from (3.6) and (3.7) that

$$
R_{T}(\mathrm{u})=\left(\frac{1}{2 \pi}\right)^{n} \int_{\mathrm{R}^{n}} e^{i(\mathrm{u}, \mathrm{s})} d F_{\mathrm{T}}(\mathrm{s})
$$

Under the condition that $\lim _{T_{1} \rightarrow \infty} \ldots \lim _{T_{n} \rightarrow \infty} R_{\mathrm{T}}(0)$ exists finitely, so that the measure defined by $F_{\mathbf{T}}(s)$ is uniformly bounded, we obtain the result of the theorem in view of Levy's continuity theorem (see Cuppens [4], p.40).

\section{ERgodicity}

The function $F(s)$ as given in Theorem 1 is defined to be the spectral distribution function of the random field $\{X(t)\}$. We now consider a mean ergodic theorem for $\{X(t)\}$ which possesses finite first and second moments:

$$
\begin{aligned}
& E X(\mathrm{t})=m(\mathrm{t}), \quad \mathrm{t} \in \mathbf{R}^{n} \\
& R(\mathrm{~s}, \mathrm{t})=E\left(X(\mathrm{~s})-m(\mathrm{~s}) \overline{(X(\mathrm{t})-m(\mathrm{t}))}, s, \mathrm{t} \in \mathbf{R}^{\mathbf{n}}\right.
\end{aligned}
$$

under a condition involving the spectral function $F(s)$ only.

Theorem 2. For a random field $\left\{X(t), t \in \mathbf{R}^{n}\right\}$ satisfying conditions (i) - (iii), and assuming $\lim _{T \rightarrow \infty} R_{T}(k)$ exists finitely for each $\mathbf{k} \in \mathbf{R}^{\mathbf{n}}$, we have

$$
\lim _{\substack{T_{j} \rightarrow \infty \\ j=1, \ldots, n}} \prod_{j=1}^{n}\left(T_{j}\right)^{-1} \int_{[0, T]}(X(t)-m(t)) d t=0
$$


in mean square if $F(\mathrm{~s})$ is continuous at $\mathrm{s}=0$.

Proof: Using the change of variables $(t, u) \rightarrow(k, t)$ with $k=u-t$ we get

$$
\begin{aligned}
E\left|\prod_{j=1}^{n}\left(T_{j}\right)^{-1} \int_{[0, T]}(X(\mathbf{t})-m(\mathrm{t})) d \mathrm{t}\right|^{2} \\
\quad=\prod_{j=1}^{n}\left(T_{j}\right)^{-2} \int_{[0, T]} \int_{[0, T]} R(\mathbf{t}, \mathbf{u}) d \mathrm{t} d \mathbf{u} \\
\quad=\prod_{j=1}^{n}\left(T_{j}\right)^{-2}\left[\left(\int_{[-T, 0]}+\int_{[0, T]}\right) \int_{a_{n}}^{b_{n}} \cdots \int_{a_{1}}^{b_{1}}\right] R(\mathbf{t}, \mathbf{t}+\mathbf{k}) d \mathrm{t} d \mathbf{k} \\
\quad=\prod_{j=1}^{n}\left(T_{j}\right)^{-1} \int_{[-T, T]} R_{\mathbf{T}}(\mathbf{k}) d \mathbf{k} \\
\quad=\prod_{j=1}^{n}\left(T_{j}\right)^{-1} \int_{\mathbb{R}^{n}} R_{\mathbf{T}}^{*}(\mathbf{k}) d \mathbf{k}
\end{aligned}
$$

where $R_{T}^{*}(\mathrm{k})$ is as defined in the proof of Lemma 2.

In view of (3.8), we have for $T_{j}, j=1, \ldots, n$, sufficiently large that

$$
\begin{aligned}
\prod_{j=1}^{n}\left(T_{j}\right)^{-1} \int_{\mathbf{R}^{n}} R_{T}^{*}(\mathrm{k}) d \mathrm{k} \\
=\left(\frac{1}{2 \pi}\right)^{n} \prod_{j=1}^{n}\left(T_{j}\right)^{-1} \int_{\mathbf{R}^{n}} \int_{\mathbf{R}^{n}} e^{i(\mathrm{u}, \mathrm{s})} d F_{\mathrm{T}}(\mathrm{s}) d \mathbf{k} \\
=\left(\frac{1}{2 \pi}\right)^{n} \prod_{j=1}^{n}\left(T_{j}\right)^{-1} \int_{\mathbf{R}^{n}} \int_{\mathbf{R}^{n}} e^{i(\mathrm{u}, \mathrm{s})} d \mathbf{k} d F_{\mathrm{T}}(\mathrm{s}) \\
\quad \text { using Lemma } 2 \text { and Fubini's theorem } \\
=\prod_{j=1}^{n}\left(T_{j}\right)^{-1} \int_{\mathbf{R}^{n}} \delta\left(s_{1}\right) \ldots \delta\left(s_{n}\right) d F_{\mathbf{T}}(\mathrm{s}),
\end{aligned}
$$

where $\delta\left(s_{i}\right)$ denotes the usual Kronecker delta function.

Under the condition that $\lim _{T \rightarrow \infty} R_{\mathrm{T}}(\mathbf{k})$ exists, Theorem 1 yields that $\lim _{T_{1} \rightarrow \infty} \cdots \lim _{T_{n} \rightarrow \infty} F_{T}(s)=F(s)$. Consequently, using Lemma 2 and the dominated con- 
vergence theorem we get

$$
\begin{aligned}
\lim _{\substack{T_{j} \rightarrow \infty \\
j=1, \ldots, n}} E\left|\prod_{j=1}^{n}\left(T_{j}\right)^{-1} \int_{[0, T]}(X(\mathrm{t})-m(\mathrm{t})) d \mathrm{t}\right|^{2} \\
=\lim _{\substack{T_{j} \rightarrow \infty \\
j=1, \ldots, n}} \prod_{j=1}^{n}\left(T_{j}\right)^{-1} \int_{T^{n}} \delta\left(s_{1}\right) \ldots \delta\left(s_{n}\right) d F(\mathrm{~s}) .
\end{aligned}
$$

It is seen that if $F(s)$ is continuous at $s=0$, the right hand side of (3.9) is zero, which gives the theorem.

\section{Appendix}

\section{Almost periodic functions on $\mathbf{R}^{\mathbf{n}}$}

A function $f \in L^{\infty}\left(\mathbf{R}^{n}\right)$ is, by definition, almost periodic if the set of all translates $\left\{f_{y} ; y \in \mathbf{R}^{n}\right\}$ of $f$ is totally bounded in the uniform norm of $L^{\infty}\left(\mathbf{R}^{n}\right)$. The space of all almost periodic functions on $\mathrm{R}^{n}$ is denoted by $A P\left(\mathrm{R}^{n}\right)$. As shown by Loomis $[7, \mathrm{p} .166]$, under the uniform norm, $A P\left(\mathbf{R}^{n}\right)$ is a commutative $C^{*}$-algebra with an identity. Also, a constant function

$$
M(f)=\int_{\mathbf{R}^{n}} f(\mathbf{x}) d \mathbf{x} \text { exists for } f \in A P\left(\mathbf{R}^{n}\right)
$$

This constant is called the mean value of $f$. It should be noted that in the above integral, $d x$ denotes the Haar measure, that is the normalised Lebesgue measure on $\mathbf{R}^{n}$ (with total mass one). We shall now prove that

$$
M(f)=\lim _{\substack{T_{j} \rightarrow \infty \\ j=1, \ldots, n}} \frac{1}{T_{1} T_{2} \ldots T_{n}} \int_{[0, T]} f(t) d(t)
$$

for $f \in A P\left(\mathbf{R}^{n}\right), \mathbf{T}=\left(T_{1}, \ldots, T_{n}\right) \in \mathbf{R}^{n}$.

Given $\varepsilon>0$, we choose the finite sets $\left\{c_{i}\right\}$ and $\left\{a_{i}\right\}$ such that $c_{i}>0, \sum c_{i}=1$ and $\left\|M(f)-\sum c_{i} f\left(\mathbf{x}-\mathbf{a}_{i}\right)\right\|_{\infty}<\varepsilon, \quad \mathbf{x}, \mathbf{a}_{i} \in \mathbf{R}^{n}$.

It follows upon integrating that

$$
\left\|M(f)-\frac{1}{T_{1} \ldots T_{n}} \sum c_{i} \int_{[0, T]} f\left(\mathbf{x}-\mathbf{a}_{i}\right) d \mathbf{x}\right\|_{\infty}<\varepsilon .
$$

By a change of variables

$$
\int_{[0, T]} f\left(x-a_{i}\right) d x=\int_{\left[-a_{i}, T-a_{i}\right]} f(t) d t .
$$


We first prove that for any $\mathbf{a}_{i}=\left(a_{i 1}, \ldots, a_{i n}\right) \in \mathbf{R}^{n}$,

$$
\lim _{\substack{T_{j} \rightarrow \infty \\ j=1, \ldots, n}} \frac{1}{T_{1} \ldots T_{n}} \int_{\left[-a_{i}, T-a_{i}\right]} f(t) d t=\lim _{\substack{T_{j} \rightarrow \infty \\ j=1, \ldots, n}} \frac{1}{T_{1} \ldots T_{n}} \int_{[0, T]} f(t) d t .
$$

For $a_{i 1} \geqslant 0$,

(A4)

$$
\begin{aligned}
& \frac{1}{T_{1} \ldots T_{n}} \int_{\left[-a_{i}, T-a_{i}\right]} f(\mathrm{t}) d \mathrm{t} \\
& \quad=\frac{1}{T_{2} \ldots T_{n}} \int_{-a_{i n}}^{T-a_{i n}} \ldots \frac{1}{T_{1}}\left(\int_{-a_{i 1}}^{0}+\int_{0}^{T_{1}}-\int_{T_{1}-a_{i 1}}^{T_{1}}\right) f(\mathrm{t}) d t_{1} \ldots d t_{n} .
\end{aligned}
$$

Now,

$$
\lim _{T_{1} \rightarrow \infty} \frac{1}{T_{1}} \int_{-a_{i 1}}^{0} f\left(t_{1}, \ldots, t_{n}\right) d t_{1}=0
$$

as $f(t)$ is bounded. Also,

$$
\begin{gathered}
\lim _{T_{1} \rightarrow \infty} \frac{1}{T_{1}} \int_{T_{1}-a_{i 1}}^{T_{1}} f\left(t_{1}, \ldots, t_{n}\right) d t_{1}=\lim _{T_{1} \rightarrow \infty} \frac{1}{T_{1}} \int_{0}^{T_{1}} f\left(t_{1}, \ldots, t_{n}\right) d t_{1} \\
-\lim _{T_{1} \rightarrow \infty} \frac{T_{1}-a_{i 1}}{T_{1}} \\
\cdot \frac{1}{T_{1}-a_{i 1}} \int_{0}^{T_{1}-a_{i 1}} f\left(t_{1}, \ldots, t_{n}\right) d t_{1} \\
=0 .
\end{gathered}
$$

Therefore, the right hand side of (A4) becomes

$$
\frac{1}{T_{2} \ldots T_{n}} \int_{-a_{\text {in }}}^{T-a_{\text {in }}} \cdots \frac{1}{T_{1}} \int_{0}^{T_{1}} f\left(t_{1}, \ldots, t_{n}\right) d t_{1} \ldots d t_{n} .
$$

A similar result holds for $a_{i 1}<0$. Repeating this process, we get (A3). It now follows from (A2) that

$$
\begin{aligned}
& \left\|M(f)-\sum c_{i} \lim _{\substack{T_{j} \rightarrow \infty \\
j=1, \ldots, n}} \frac{1}{T_{1} \ldots T_{n}} \int_{[0, T]} f(\mathrm{t}) d \mathrm{t}\right\|_{\infty} \\
& \quad=\left\|M(f)-\lim _{\substack{T_{j} \rightarrow \infty \\
j=1, \ldots, n}} \frac{1}{T_{1} \ldots T_{n}} \int_{[0, T]} f(\mathrm{t}) d \mathrm{t}\right\|_{\infty} \\
& <\varepsilon .
\end{aligned}
$$

Since $\varepsilon$ can be arbitrarily small, we get

$$
M(f)=\lim _{\substack{T_{j} \rightarrow \infty \\ j=1, \ldots, n}} \frac{1}{T_{1} \ldots T_{n}} \int_{[0, T]} f(\mathrm{t}) d \mathrm{t}
$$




\section{REFERENCES}

[1] C.S.K. Bhagavan, Non-stationary processes, spectra and some ergodic theorems (Andhra University Press, 1974).

[2] C.S.K. Bhagavan, 'A mean ergodic theorem for a class of continuous parameter non-stationary processes', Sankhyā Ser. A 37 (2) (1975), 292-296.

[3] C.S.K. Bhagavan, 'On nonstationary time series', in Handbook of statistics 5, E.J. Hannan, P.R. Krishnaiah, M.M. Rao (Elsevier, 1985).

[4] R. Cuppens, Decomposition of Multivariate Probabilities (Academic Press, 1975).

[5] J. Kampe de Feriet and F.N. Frenkiel, 'Correlations and spectra for non-stationary random functions', Math. Comp. 16 (1962), 1-21.

[6] M. Loeve, 'Fonctions aleatoires du second ordre. Supplement to P. Levy', Processus Stochastiques et Mouvement Brownien, (Paris, 1948).

[7] L.H. Loomis, An Introduction to Abstract Harmonic Analysis (Van Nostrand, 1953).

[8] E. Parzen, 'Spectral analysis of asymptotically stationary time series', Bull. Inst. Internat. Statist. 39 (1962), 87-103.

[9] M.M. Rao, 'Covariance analysis of non-stationary time series', in Developments in Statistics 1, ed. P.R. Krishnaiah, pp. 171-225 (Academic Press, 1978).

[10] M.M. Rao, 'Harmonizable, Cramer and Karhunen classes of processes', in Handbook of statistics 5, Editors E.J. Hannan, P.R. Krishnaiah, M.M. Rao (Elsevier, 1985).

[11] J. Wolters, Stochastic dynamic properties of linear econometric models (Springer Verlag, Berlin, Heidelberg, New York, 1980).

School of Mathematics

Queensland University of Technology

GPO Box 2434

Brisbane Qld 4001

Australia 